\title{
A Relação entre a Atenção Compartilhada e a Teoria da Mente: Um Estudo Longitudinal
}

\author{
The Relationship between Joint Attention and Theory of Mind: \\ A Longitudinal Study
}

\author{
Camila Soares de Abreu* ${ }^{*}$, Cláudia Cardoso-Martins ${ }^{a} \&$ Poliana Gonçalves Barbosa $^{b}$ \\ ${ }^{a}$ Universidade Federal de Minas Gerais, Belo Horizonte, Minas Gerais, Brasil \\ $\&{ }^{b}$ Universidade de Alberta, Edmonton, Província de Alberta, Canadá
}

\begin{abstract}
Resumo
Este estudo avaliou a relação entre a habilidade de a criança compartilhar sua atenção com a atenção de outras pessoas e o desenvolvimento posterior da teoria da mente (i.e., a compreensão de que as pessoas possuem estados mentais como, por exemplo, desejos, intenções e crenças), em uma amostra de 28 crianças de famílias de classe média-baixa/média-alta. Os resultados mostraram que a habilidade de as crianças seguirem o gesto de olhar ou apontar do examinador aos nove meses de idade predisse significativamente seu desempenho em tarefas de crença-falsa aos quatro anos, independentemente de variações na inteligência não verbal. Esses resultados sugerem que, desde o final do primeiro ano de vida, as crianças interpretam o gesto de olhar/apontar das pessoas ao seu redor como ações intencionais.

Palavras-chave: Atenção compartilhada, teoria da mente, tarefas de crença-falsa.
\end{abstract}

\begin{abstract}
We investigated the relationship between infants' ability to coordinate their attention with that of other people and later Theory of Mind skills (i.e., the understanding that people have mental states such as desires, intentions and beliefs) in a sample of 28 children from middle class families in Brazil. Results showed that children's ability to follow the examiner's gaze and pointing gestures at nine months of age significantly predicted their performance on false-belief tasks at the age of four years, independently of variations in their non-verbal intelligence. These findings suggest that, at end of the first year of life, children's joint attention behaviors indicate intentional understanding. Keywords: Joint attention, theory of mind, false-belief tasks.
\end{abstract}

Em torno do final do primeiro ano de vida, observa-se uma mudança radical nas interações que os bebês estabelecem com as pessoas ao seu redor. Até então essencialmente caracterizadas por intercâmbios face-a-face, as interações entre os bebês e as outras pessoas passam a incorporar, de forma ativa e coordenada, a atenção a objetos e eventos no meio ambiente. Por exemplo, por volta dos 9-12 meses de idade, os bebês começam a olhar na direção do gesto de olhar ou de apontar de um adulto buscando, aparentemente, identificar o foco da sua atenção. Eles também começam a mostrar objetos para as outras pessoas verem. Esses comportamentos têm sido denominados de comportamentos de atenção compartilhada ou conjunta e, segundo um ponto de vista proeminente na literatura (ver, e.g., Tomasello \& Carpenter, 2005), prenunciam o nascimento da "teoria da mente", ou seja, da compreensão de que as pessoas são seres psicológicos, cujas ações ou comportamentos são determinados por estados mentais. Com efeito, ao voltar seus olhos na direção do olhar de outra pessoa, os bebês denotam compreender o olhar como um ato proposital que envolve a percepção de um objeto ou evento particular no meio ambiente.

O desenvolvimento da teoria da mente tem sido o foco de inúmeros estudos nos últimos anos (Flavell, 2007;
*Endereço para correspondência: Faculdade de Filosofia e Ciências Humanas, Laboratório de Desenvolvimento Cognitivo e da Linguagem, Universidade Federal de Minas Gerais, Av. Antônio Carlos, 6.627, Sala 2.000, Caixa Postal No 253, Belo Horizonte, MG, Brasil 31270-901. E-mail: casoares.abreu@yahoo.com.br

Este estudo é parte de um projeto de pesquisa sobre o desenvolvimento da habilidade de comunicação verbal e não-verbal em crianças falantes do português e inglês.
Sua realização foi possível graças a um auxilio financeiro do Conselho Nacional de Desenvolvimento Científico e Tecnológico (CNPq) e da Fundação de Amparo à Pesquisa do Estado de Minas Gerais (FAPEMIG) à segunda autora, e a uma bolsa de Mestrado concedida à primeira autora pelo CNPq. As autoras agradecem as famílias que participaram do estudo, à professora Catharine Echols pela colaboração, entusiasmo e apoio constantes e, finalmente, a Virgínia Nicolau Gonçalves e André Luiz Souza, pela ajuda na coleta e análise dos dados. 
Peterson, Wellman, \& Liu, 2005; Souza, 2008; Wellman, Cross, \& Watson, 2001). Em particular, um número grande de estudiosos tem investigado o desenvolvimento da compreensão pela criança de que as crenças são representações mentais e, como tal, podem ou não corresponder à realidade. Essa compreensão é comumente avaliada através de tarefas de "crença-falsa", das quais a tarefa de Sally e Ann constitui um exemplo prototípico (Baron-Cohen, Leslie, \& Frith, 1985). A tarefa desenrola-se da seguinte maneira:

Sally tem uma bola de gude e uma caixa; Ann tem uma cesta. Sally coloca a bola de gude na caixa e sai para dar um passeio. Na sua ausência, Ann pega a bola e a coloca em sua cesta. Sally retorna do passeio e quer brincar com sua bola de gude. Onde Sally irá buscar a bola?

A julgar pelos resultados de inúmeros estudos, é apenas em torno dos 4-5 anos de idade que a maioria das crianças responde que Sally irá buscar a bola onde acredita que ela esteja, ou seja, na caixa. Antes dessa idade, as crianças aparentemente não compreendem que as crenças nem sempre correspondem à realidade ou que duas pessoas possam ter representações diferentes acerca do mundo real. Embora se lembrem perfeitamente do local onde Sally escondeu a bola, elas respondem que Sally irá procurar pela bola no local em que esta se encontra de verdade (Wellman et al., 2001; Wimmer \& Perner, 1983; mas ver Onish \& Baillargeon, 2005, para uma visão diferente).

A compreensão de crenças falsas é considerada um marco importante no desenvolvimento da teoria da mente. Com efeito, alguns filósofos (e.g., Dennett, 1987) têm argumentado que essa compreensão é a única evidência inequívoca de que as crianças são capazes de imputar estados mentais a outras pessoas. Não obstante, e a despeito do enorme interesse que a teoria da mente tem despertado entre pesquisadores do desenvolvimento humano, poucos estudos têm investigado seus precursores no desenvolvimento inicial da criança. No presente estudo, investigamos a hipótese de que a habilidade de atenção compartilhada constitui um precursor importante da compreensão de crenças falsas.

Vários tipos de evidência conspiram a favor dessa hipótese. É notável, por exemplo, que crianças autistas apresentem déficits acentuados tanto na habilidade de atenção compartilhada (Bruinsma, Koegel, \& Koegel, 2004; Charman, 2003; Charman et al., 1997) quanto na compreensão de crenças falsas (Baron-Cohen et al., 1985; Perner, Frith, Leslie, \& Leekam, 1989; Peterson et al., 2005). Da mesma maneira, a evidência de uma associação entre a atenção compartilhada e o desenvolvimento da linguagem sugere que a criança atribui intenção comunicativa aos comportamentos de atenção compartilhada (Bosa, 2002; Carpenter, Nagell, \& Tomasello, 1998; Morales et al., 2000; Tomasello \& Farrar, 1986). Contudo, como esses exemplos demonstram, a maior parte da evidência de que dispomos é indireta. Ao que tudo indica, apenas três estudos (Charman et al., 2000; McLean, 2009; Nelson, Adamson, \& Bakeman, 2008) investigaram diretamente a relação entre a atenção compartilhada e o desenvolvimento posterior da teoria da mente.

Charman et al. (2000) investigaram a relação entre a atenção compartilhada e o desenvolvimento posterior da teoria da mente em uma amostra de 13 crianças. De acordo com os autores, a atenção compartilhada, avaliada quando as crianças tinham 20 meses de idade, correlacionou-se significativamente com medidas de teoria da mente aos 44 meses de idade.

No estudo de Charman et al. (2000), a avaliação da teoria da mente não incluiu tarefas de crença-falsa, mas habilidades que desenvolvem-se mais precocemente como, por exemplo, a compreensão de que, dependendo de sua posição em relação à criança, uma outra pessoa pode ter uma visão diferente de um mesmo objeto ou cenário. No estudo de Nelson et al. (2008), por outro lado, a teoria da mente foi avaliada através de tarefas de crença-falsa.

Quarenta e duas crianças e suas mães participaram do estudo de Nelson et al. (2008). A atenção compartilhada foi avaliada entre 18 e 30 meses de idade, em termos da habilidade de a criança monitorar ou chamar a atenção da mãe para um objeto através de vocalizações e/ou gestos (e.g., apontando para um objeto ou simplesmente alternando seu olhar entre o objeto e a mãe). A teoria da mente foi avaliada posteriormente, entre 42 e 66 meses de idade, através de duas tarefas de crença-falsa. Os resultados mostraram uma associação significativa entre a habilidade de atenção compartilhada e o desempenho nas tarefas de teoria da mente, mesmo após Nelson et al. (2008) terem controlado o efeito de diferenças na inteligência verbal das crianças. Resultados semelhantes foram encontrados por McLean (2009) em um grupo de crianças em risco de virem a apresentar um transtorno do espectro autista.

McLean (2009) avaliou a relação entre a atenção compartilhada e o desempenho em tarefas de teoria da mente, incluindo tarefas de crença falsa em dois grupos de crianças: um grupo de crianças que tinham um irmão ou irmã com o diagnostico de autismo (grupo de risco) e um grupo de crianças cujos irmãos ou irmãs estavam desenvolvendo-se tipicamente (grupo sem risco). De acordo com McLean (2009), a atenção compartilhada, avaliada quando as crianças tinham 12 meses de idade predisse o desenvolvimento da teoria da mente aos 5 anos de idade, mas apenas entre as crianças do grupo de risco.

O presente estudo é mais uma tentativa de avaliar a relação entre a atenção compartilhada e o desenvolvimento posterior da compreensão de crenças falsas. Nosso estudo distingue-se dos estudos anteriores (Charman et al., 2000; McLean, 2009; Nelson et al., 2008) em dois aspectos importantes. Em primeiro lugar, a atenção compartilhada foi avaliada quando as crianças tinham apenas 9 meses de idade, ou seja, na idade em que essa habilidade começa a despontar no comportamento dos bebês. Em segundo lugar, ao contrário dos estudos anteriores, que avaliaram apenas a habilidade de a criança iniciar (e.g., a criança aponta para um objeto) ou responder (e.g., a criança olha 
Abreu, C. S., Cardoso-Martins, C. \& Barbosa, P. G. (2014). A Relação entre a Atenção Compartilhada e a Teoria da Mente: Um Estudo Longitudinal.

na direção do gesto de apontar de outra pessoa) à atenção compartilhada, o presente estudo incluiu medidas de ambas as habilidades.

Ao contrário do que parece ocorrer em relação aos comportamentos de iniciar atenção compartilhada, a interpretação do comportamento infantil de seguir o gesto de olhar ou apontar das outras pessoas tem sido objeto de controvérsia na literatura. Em oposição à interpretação "rica" de Tomasello (e.g., Tomasello \& Carpenter, 2005) que argumenta que os comportamentos de atenção compartilhada denotam uma compreensão implícita de intencionalidade, alguns autores têm sugerido que os comportamentos de responder à atenção compartilhada podem ser explicados em termos de uma aprendizagem condicionada. Por exemplo, é possível que os bebês simplesmente aprendam que o gesto de olhar ou apontar de um adulto é, de modo geral, seguido de um objeto ou evento interessante no prolongamento exato daquele gesto (e.g., Moore $\&$ Corkum, 1994). Ao que tudo indica, apenas o estudo de McLean (2009) avaliou a relação entre a habilidade de responder à atenção compartilhada e o desenvolvimento da teoria da mente. Contudo, esse estudo incluiu uma amostra muito pequena de crianças (14 crianças sem risco e 9 crianças com risco). Além disso, conforme observamos anteriormente, seus resultados foram pouco conclusivos. A avaliação da habilidade de responder à atenção compartilhada no presente estudo poderá, portanto, contribuir para esclarecer essa questão.

\section{Método}

\section{Participantes}

Vinte e oito crianças (22 meninas, 6 meninos) provenientes de famílias de classe socioeconômica média-baixa/ média-alta participaram do estudo. As crianças foram recrutadas com o auxílio de pediatras na cidade de Belo Horizonte, MG, um pouco antes de completaram 9 meses de idade. Apenas crianças nascidas a termo e que, segundo as mães e os pediatras, estavam se desenvolvendo tipicamente, foram incluídas no estudo. Conforme ilustrado na Tabela 1, todas as crianças apresentaram um desempenho normal ou acima do normal em um teste de inteligência não-verbal, administrado quando elas tinham, em média, 3 anos e 7 meses de idade ( $D P=1,3$ meses). As mães de todas as crianças assinaram um termo de consentimento livre e esclarecido.

\section{Procedimentos}

O presente estudo é parte de um estudo longitudinal sobre o desenvolvimento inicial da habilidade de comunicação verbal e não-verbal em português. No presente trabalho, relatamos os resultados de análises que investigaram a relação entre a habilidade de atenção compartilhada, avaliada quando a criança tinha 9 meses de idade, e a compreensão de crenças falsas, avaliada aos 4 anos e 2 meses de idade. A inteligência não-verbal foi avaliada quando a criança tinha 3 anos e 7 meses de idade.
As avaliações ocorreram na casa da criança, em dia e horário determinados pelas mães. Todas foram filmadas para posterior verificação.

\section{Instrumentos de Avaliação}

Atenção Compartilhada. A Forma Abreviada das Escalas de Comunicação Social Inicial (Mundy et al., 2003) foi utilizada para avaliar a habilidade de a criança iniciar e responder à atenção compartilhada. Para a avaliação, a criança foi colocada no colo da mãe, diante de uma mesa e de frente para o examinador, sendo a mãe instruída a não interagir com a criança ou estimulá-la. A administração da escala durou aproximadamente 20 minutos.

Os comportamentos de atenção compartilhada são divididos entre comportamentos de nível inferior e de nível superior. Os comportamentos de iniciar atenção compartilhada de nível inferior incluem "alternar o olhar entre um objeto (e.g., um brinquedo mecânico acionado pelo examinador) e o examinador" e "olhar para o examinador ao manipular um brinquedo". Os seguintes comportamentos de iniciar atenção compartilhada de nível superior são incluídos na escala: "mostrar um brinquedo para o examinador, estendendo o braço na sua direção", "apontar na direção de gravuras afixadas na parede", "apontar para figuras em um livro" e, finalmente, "apontar para um brinquedo mecânico em movimento". Para cada nível, o escore da criança equivale ao número de vezes em que ela evidencia esses comportamentos durante a avaliação.

A habilidade de responder à atenção compartilhada de nível inferior e superior corresponde à habilidade de a criança seguir com o olhar o gesto do examinador de apontar para gravuras em um livro e gravuras afixadas na parede, respectivamente. Para cada nível, o escore da criança corresponde à proporção de respostas corretas (de um total de oito, para a habilidade de responder à atenção compartilhada de nível inferior e de um total de seis, para a habilidade de responder à atenção compartilhada de nível superior), multiplicado por 100 . O escore total consiste na soma dos dois escores individuais (Escore Máximo $=200$ ).

Com o objetivo de avaliar a confiabilidade das escalas de atenção compartilhada na presente amostra, as respostas das 28 crianças foram classificadas por dois juízes independentes. $\mathrm{O}$ índice de confiabilidade, calculado como o número de acordos dividido pelo número de acordos somados ao de desacordos $\left[\mathrm{n}^{\circ}\right.$ de acordos $/\left(\mathrm{n}^{\circ}\right.$ de acordos $+\mathrm{n}^{\mathrm{o}}$ de desacordos)], foi 0,70 para a habilidade de iniciar atenção compartilhada e 0,77 , para a habilidade de responder à atenção compartilhada.

Teoria da Mente. Cinco tarefas, semelhantes à tarefa de Sally e Ann descrita anteriormente foram utilizadas para avaliar a compreensão de crenças-falsas quando as crianças estavam com 50 meses de idade. Além da questão-alvo (e.g., "Onde Sally vai procurar sua bola?"), a criança devia responder a uma ou duas questões de controle (e.g., "Onde a bola está de verdade?"; "Onde Sally colocou a bola antes de sair para passear?"). Todas as questões deviam ser respondidas corretamente para a criança receber crédito 
na tarefa. $\mathrm{O}$ número de tarefas respondidas corretamente é computado.

Apenas tarefas frequentemente descritas na literatura (e.g., Domingues, Valério, Panciera, \& Maluf, 2007) foram usadas no presente estudo. Uma descrição detalhada das tarefas poderá ser obtida do primeiro autor.

A Escala de Maturidade Mental Columbia (Alves \& Duarte, 1993) foi utilizada para avaliar a inteligência não-verbal quando as crianças tinham 43 meses de idade. O teste avalia a habilidade de a criança identificar, entre três e cinco figuras de objetos (e.g., cama, armário, escrivaninha, janela) aquela que é "diferente" ou "não combina" com as demais (a figura da janela no exemplo acima). $\mathrm{O}$ teste consiste de 92 itens, mas apenas os itens correspondentes à idade cronológica da criança são administrados. No presente estudo, as crianças foram submetidas aos 55 itens recomendados para a faixa etária compreendida entre 3 anos e 6 meses e 3 anos e 11 meses. $\mathrm{O}$ resultado bruto obtido (i.e., o número de respostas corretas), é convertido em um índice de maturidade mental. A adaptação brasileira apresenta índices apropriados de confiabilidade interna $(0$, 87 ) e confiabilidade teste-reteste $(0,80)$.

\section{Resultados}

A Tabela 1 lista os escores médios e o desvio padrão para as medidas de atenção compartilhada, teoria da mente e para o teste de inteligência. Tendo em vista a baixa frequência de comportamentos de atenção compartilhada de "nível superior" (apenas três e oito crianças, respectivamente, apresentaram comportamentos de iniciar e responder à atenção compartilhada de nível superior), para as análises descritas a seguir, os escores de "nível inferior" e "nível superior" foram combinados em um único escore. Os índices de correlação entre as diversas habilidades avaliadas aparecem na Tabela 2.

Tabela 1

Escore Médio (e desvio padrão) para as Medidas de Atenção Compartilhada, Teoria da Mente e Columbia

\begin{tabular}{lccc}
\hline Medidas & Mínimo & Máximo & Média $(D P)$ \\
\hline IAC (ni) $)^{\mathrm{a}}$ & 3 & 16 & $7,36(3,63)$ \\
IAC (ns) & 0 & 1 & $0,07(0,26)$ \\
IAC (total) & 3 & 16 & $7,43(3,62)$ \\
RAC (ni) $($ Max = 100) & 0 & 67 & $26,14(20,98)$ \\
RAC (ns) $($ Max = 100) & 0 & 38 & $7,21(12,10)$ \\
RAC (total) $($ Max =200) & 0 & 104 & $33,32(28,75)$ \\
Columbia & 4,0 & 9,0 & $6,03(1,30)$ \\
ToM (Max $=6)$ & 0 & 5 & $2,46(1,20)$ \\
\hline
\end{tabular}

Nota. $N=28 ; \mathrm{IAC}=$ Iniciar atenção compartilhada; $\mathrm{RAC}=$ responder à atenção compartilhada; ni= nível inferior; ns= nível superior; $\mathrm{ToM}=$ teoria da mente.

${ }^{\mathrm{a} N u m e r o}$ de respostas apresentadas durante a avaliação; ${ }^{\mathrm{b}}$ idade mental em anos.

Tabela 2

Correlações entre as Habilidades de Atenção Compartilhada, as Tarefas de Teoria da Mente, e a Escala de Maturidade Mental Columbia

\begin{tabular}{lcccc}
\hline & IAC & RAC & Col & ToM \\
\hline IAC & & 0,15 & 0,11 & 0,02 \\
RAC & & & $0,57^{*}$ & $0,59^{*}$ \\
Col & & & & $0,54^{*}$
\end{tabular}

ToM

Nota. $N=28 ;$ IAC = iniciar atenção compartilhada; RAC $=$ responder à atenção compartilhada; ToM = teoria da mente. $* p<0,01$.
Conforme ilustrado na Tabela 2, a habilidade de responder à atenção compartilhada, mas não a habilidade de iniciar atenção compartilhada, correlacionou-se significativamente com o desempenho nas tarefas de teoria da mente aos 4 anos e 2 meses. Esses resultados são aparentemente consistentes com hipótese de que os comportamentos de atenção compartilhada refletem as origens de uma teoria da mente. Contudo, é possível que a relação observada entre a atenção compartilhada e as medidas de teoria da mente tenha sido mediada por variações na inteligência da criança. Com efeito, o desempenho na Escala de Maturidade Mental Columbia correlacionou-se tanto com a habilidade de responder à atenção compartilhada, quanto com os escores das crianças nas tarefas de teoria da mente. Em vista disso, uma análise de regressão hierárquica foi realizada com 
Abreu, C. S., Cardoso-Martins, C. \& Barbosa, P. G. (2014). A Relação entre a Atenção Compartilhada e a Teoria da Mente: Um Estudo Longitudinal.

o objetivo de controlar o efeito de variações na inteligência das crianças. Para tanto, os escores no teste de Colúmbia entraram na equação no primeiro passo, ou seja, antes dos escores correspondentes às habilidades de responder à atenção compartilhada. Como pode ser visto na Tabela 3 , a habilidade de responder à atenção compartilhada continuou a contribuir significativamente para o desempenho nas tarefas de crença falsa, mesmo após o controle do efeito de diferenças na inteligência não-verbal.

Tabela 3

Regressões Hierárquicas: A Contribuição da Habilidade de Responder à Atenção Compartilhada para o Desenvolvimento da Teoria da Mente

\begin{tabular}{lcc}
\hline $\begin{array}{l}\text { Variáveis } \\
\text { independentes }\end{array}$ & \multicolumn{2}{c}{ Teoria da mente } \\
\cline { 2 - 3 } & $R$ & $\Delta R^{2}$ \\
\hline $1^{\circ}$ passo: & 0,544 & $0,296^{* *}$ \\
Columbia & & \\
$2^{\circ}$ passo: & 0,638 & $0,111^{*}$ \\
RAC &
\end{tabular}

Nota. $\mathrm{RAC}=$ responder à atenção compartilhada. $* p<0,05 ; * * p<0,01$.

\section{Conclusões}

No presente estudo, avaliamos a correlação entre a habilidade de atenção compartilhada aos 9 meses de idade e o desenvolvimento da teoria da mente aos 4 anos e 2 meses. Tanto a habilidade de iniciar quanto a habilidade de responder à atenção compartilhada foram investigadas. Os resultados mostraram que a habilidade de responder à atenção compartilhada, mas não a habilidade de iniciar a atenção compartilhada, correlacionou-se significativamente com o desenvolvimento posterior da teoria da mente, mesmo após o controle do efeito de variações na inteligência da criança.

Nos estudos que investigaram a relação entre a habilidade de atenção compartilhada e o desenvolvimento posterior da teoria da mente (Charman et al., 2000; McLean, 2009; Nelson et al., 2008), a atenção compartilhada foi avaliada quando as crianças tinham entre 12 e 30 meses de idade, ou seja, em uma idade em que essa habilidade possivelmente já está bem desenvolvida. Um diferencial importante do nosso estudo é que as crianças tinham apenas 9 meses de idade quando a atenção compartilhada foi avaliada. Nossos resultados estendem, portanto, os resultados daqueles estudos e sugerem que, desde as suas primeiras manifestações, o comportamento de seguir o olhar ou o gesto de apontar do adulto reflete a compreensão de que as pessoas são agentes ativos, cujos gestos ou ações possuem intenção comunicativa.
Em oposição aos resultados encontrados para os comportamentos de responder à atenção compartilhada, não encontramos uma associação entre os comportamentos de iniciar atenção compartilhada e o desempenho nas tarefas de teoria da mente. Esses resultados foram inesperados. Ao contrário do comportamento de olhar na direção do olhar ou do gesto de apontar do adulto, interpretado como uma resposta condicionada por alguns pesquisadores (e.g., Moore \& Corkum, 1994), não parece haver dúvida de que os comportamentos de iniciar atenção compartilhada refletem a tentativa da criança de influenciar o estado mental das outras pessoas. Com efeito, nos estudos de Charman et al. (2000) e Nelson et al. (2008) foram precisamente esses comportamentos que predisseram o desenvolvimento posterior da teoria da mente. Como então explicar os resultados negativos encontrados para a habilidade de iniciar atenção compartilhada no presente estudo?

É pouco provável que esses resultados possam ser explicados em termos de uma restrição na variação dos comportamentos de iniciar a atenção compartilhada. Como pode ser observado na Tabela 1, assim como ocorreu para os comportamentos de responder à atenção compartilhada, a frequência dos comportamentos de iniciar a atenção compartilhada variou consideravelmente entre as crianças que participaram do presente estudo. É possível, no entanto, que a habilidade de iniciar a atenção compartilhada das crianças que participaram do presente estudo fosse ainda muito incipiente. De fato, praticamente não observamos exemplos do que Mundy et al. (2003) denominaram de comportamentos de iniciar atenção compartilhada de nível superior (e.g., a criança aponta para um brinquedo mecânico em movimento e para gravuras na parede ou, então, eleva os braços de forma a exibir um objeto para o examinador). A maior parte dos comportamentos de iniciar a atenção compartilhada consistiu de respostas de alternar o olhar entre um brinquedo mecânico em movimento e o examinador, em um contexto em que este estava claramente engajado com a criança e o objeto de sua atenção. Não é certo, portanto, que a criança estivesse tentando influenciar o estado atencional do examinador.

Uma limitação do presente estudo diz respeito ao tamanho relativamente pequeno da amostra. Claramente, novos estudos, envolvendo uma amostra mais representativa de crianças, são necessários para avaliar a generalidade de nossos resultados. Do ponto de vista positivo, por outro lado, nosso estudo é aparentemente o primeiro na literatura a demonstrar uma associação entre comportamentos de responder à atenção compartilhada aos 9 meses de idade e o desenvolvimento posterior da habilidade de refletir explicita e conscientemente sobre estados mentais. Esses resultados questionam a hipótese de que a habilidade de seguir o gesto de olhar ou de apontar das outras pessoas é o resultado de uma aprendizagem condicionada (Moore \& Corkum, 1994). Ao invés disso, eles sugerem que, desde a sua origem no final do primeiro ano de vida, os comportamentos de responder à atenção compartilhada refletem a compreensão pela criança de 
que as pessoas são agentes intencionais (e.g., Tomasello \& Carpenter, 2005).

\section{Referências}

Alves, I. C. B., \& Duarte, J. L. M. (1993). Padronização brasileira da Escala de Maturidade Mental Colúmbia. In B. B. Burgmeister, L. H. Blum, \& I. Lorge (Eds.), Escala de Maturidade Mental Colúmbia: Manual para aplicação e interpretação (3. ed., pp. 25-35). São Paulo, SP: Casa do Psicólogo.

Baron-Cohen, S., Leslie, A. M., \& Frith, U. (1985). Does the autistic children have a theory of mind? Cognition, 21, 37-46.

Bosa, E. (2002). Atenção compartilhada e identificação precoce do autismo. Psicologia: Reflexão e Crítica, 15(1), 77-88.

Bruinsma, Y., Koegel, R. L., \& Koegel, L. K. (2004). Joint attention and children with autism: A review of the literature. Mental Retardation and Developmental Disabilities Research Reviews, 10, 169-175.

Carpenter, M., Nagell, K., \& Tomasello, M. (1998). Social cognition, joint attention, and communicative competence from 9 to 15 months of age. Monographs of the Society for Research in Child Development, 63(4, Serial No. 255), i-vi, 1-174.

Charman, T. (2003). Why is joint attention a pivotal skill in autism? Philosofical Transactions of the Royal Society B, 358, 315-324.

Charman, T., Baron-Cohen, S., Swettenham, J., Baird, G., Cox, A., \& Drew, A. (2000). Testing joint attention, imitation, and play as infancy precursors to language and theory of mind. Cognitive Development, 15, 481-498.

Charman, T., Swettenham, J., Baron-Cohen, S., Cox, A., Baird, G., \& Drew, A. (1997). Infants with autism: An investigation of empathy, pretend play, joint attention, and imitation. Developmental Psychology, 33(5), 781-789.

Dennett, D. C. (1987). The intentional stance. Cambridge, MA: Bradford Book

Domingues, S. F. S., Valério, A., Panciera, S. D. P., \& Maluf, M. R. (2007). Tarefas de crença falsa na avaliação de atribuição de estados mentais de crença. In P. W. Shelini (Ed.), Alguns domínios da avaliação psicológica (pp. 141-162). Campinas, SP: Alínea.

Flavell, J. (2007). Theory-of-mind development. In G. W. Ladd (Ed.), Appraising the human developmental sciences: Essays in honor of Merrill-Palmer Quartelly (pp. 38-55). Detroit, MI: Wayne State University Pres.

McLean, L. (2009). The relation between early joint attention responding and later theory of mind in younger siblings of children with autism. Retrieved September 20, 2012, from http://discoverarchive.vanderbilt.edu/handle/1803/2964

Moore, C., \& Corkum, V. (1994). Social understanding at the end of the first year of life. Developmental Review, 14, 349-372.

Morales, M., Mundy, P., Delgado, C. E. F., Yale, M., Messinger, D., Neal, R., \& Scwhartz, H. K. (2000). Responding to joint attention across the 6- to 24-month age period and early language acquisition. Journal of Applied Developmental Psychology, 21(3), 283-298.

Mundy, P., Delgado, C., Block, J., Venezia, M., Hogan, A., \& Seibert, J. (2003). A Manual for the abridged early social communication scales (ESCS). Retrieved February 15, 2009, from http://www.ucdmc.ucdavis.edu/mindinstitute/ourteam/ faculty_staff/escs.pdf

Nelson, P. B., Adamson, L. B., \& Bakeman, R. (2008). Toddler's joint engagement experience facilitates prescholers' acquisition of theory of mind. Developmental Science, 11, 847-852.
Onish, K. H., \& Baillargeon, R. (2005). Do 15 Month-old infants understand false-beliefs? Science, 308, 255-258.

Perner, J., Frith, U., Leslie, A. M., \& Leekam, S. R. (1989). Exploration of the autistic child's theory of mind: Knowledge, belief, and communication. Child Development, 60(3), 689-700.

Peterson, C. C., Wellman, H., \& Liu, D. (2005). Steps in theoryof-mind development for children with deafness or autism. Child Development, 76(2), 502-517.

Souza, D. H. (2008). De onde pra onde? As interfaces entre linguagem, teoria da mente e desenvolvimento social. In T. M. Sperb \& M. R. Maluf(Eds.), Desenvolvimento sociocognitivo: Estudos brasileiros sobre teoria da mente (pp. 33-54). São Paulo, SP: Vetor.

Tomasello, M., \& Carpenter, M. (2005). The emergence of social cognition in three young chimpanzees. Monographs of the Society for Research in Child, 70(1), vii-132.

Tomasello, M., \& Farrar, J. M. (1986). Joint attention and early language. Child Development, 57(6), 1454-1463.

Wellman, H. M., Cross, D., \& Watson, J. (2001). Meta-analysis of theory-of-mind development: The truth about false-belief. Child Development, 72, 655-684.

Wimmer, H., \& Perner, J. (1983). Beliefs about beliefs: Representation and constraining function of wrong beliefs in young children's understanding of deception. Cognition, 13, 103-128. 